\section{Clinical and Pathological Factors Related to 18F-FDG-PET Positivity in the Diagnosis of Recurrence and/or Metastasis in Patients with Differentiated Thyroid Cancer}

\section{TO THE EDITORS:}

I read with interest the article by D. Esteva and coworkers. ${ }^{1}$ The authors present a concise analysis of the diagnostic test performance of ${ }^{18} \mathrm{~F}$-FDG-PET in detecting recurrence and/or metastasis in 50 thyroid cancer patients with a negative ${ }^{131} \mathrm{I}$ whole-body scan and elevated serum thyroglobulin. The association between various clinical/ tumor-related factors and ${ }^{18}$ F-FDG-PET positivity was analyzed. While the study methodology was appropriate and well described, we feel there were some important aspects of the statistical analysis and interpretation that require discussion.

In the design section it is stated "Student's $t$ test ... was used for the statistical analysis...." This test relies on the assumption of the data being continuous and adhering to a normal distribution and essentially evaluates the strength of the evidence to support a difference in means between the 2 groups being compared. The authors compared various clinical and tumor variables between ${ }^{18} \mathrm{~F}$-FDG-PET positive and negative patients. However, 4 of the 11 explanatory variables were binary (sex, thyroid capsular invasion, lymph-node involvement, multifocality) and 1 variable was ordinal categorical (OHIO score). The comparison of these variables would be more appropriate using a test of the difference in the proportions such as a chi- square test. Furthermore, the dataset for the variables is relatively small and a nonparametric test (testing for a difference in medians) such as the Mann-Whitney $U$ test may be more valid. Generally speaking, a sample size of at least 30 in each group would be considered necessary to use parametric statistical tests. ${ }^{2}$

Positive and negative likelihood ratios are appropriate ways to express the performance of a diagnostic test that are independent of the disease prevalence in the underlying study population. However, in the results section, the authors state that "PLR [positive likelihood ratio] $>1$ indicates that the test supports a definitive diagnosis." This statement is misleading. Using a more accepted interpretation, PLR $<2$ conveys a small increment in raising the post-test probability of having the disease in question, PLR $2-5$ conveys a modest but potentially important change, PLR 5-10 conveys a moderate improvement, and PLR $>10$ indicates a large, more conclusive change in post-test probability. ${ }^{2}$

Kevin London, BHB, DipSci, MBChB, DCH, FRACP, MM, and Michael Lin, FRCP

Department of Nuclear Medicine and PET, Liverpool Hospital, Liverpool, Sydney, Australia

e-mail: kevin.london@sswahs.nsw.gov.au

Published Online: 27 May 2010

(C) Society of Surgical Oncology 2010

\section{REFERENCES}

1. Esteva D, Muros MA, Llamas-Elvira JM, Jimenez-Alonso J, Villar JM, Lopez de la Torre M, et al. Clinical and pathological factors related to 18F-FDG-PET positivity in the diagnosis of recurrence and/or metastasis in patients with differentiated thyroid cancer. Ann Surg Oncol. 2009;16:2006-13.

2. Peat JK, Barton B. Medical statistics: a guide to data analysis and critical appraisal. 1st ed. Malden, MA: Blackwell Publishing; 2005. 